Nota

\title{
INFLUÊNCIA DO TEMPO DE AGITAÇÃO NA ADSORÇÃO DE Cd, Cu, Ni e Zn EM LATOSSOLO TRATADO COM LODO DE ESGOTO (1)
}

\author{
ESTÊVÃO VICARI MELLIS $\left(\left(^{*}\right)\right.$; ARNALDO ANTÔNIO RODELLA $\left(^{3}\right)$
}

\begin{abstract}
RESUMO
O objetivo deste trabalho foi avaliar o efeito do tempo de agitação no equilíbrio de adsorção em amostra de um Latossolo não tratado e tratado com lodo de esgoto da estação de tratamento (ETE) de Barueri, coletadas na camada 0-0,2 m. A influência do tempo de agitação na adsorção foi avaliada através da adição de $20 \mathrm{~mL}$ do eletrólito suporte $\mathrm{Ca}\left(\mathrm{NO}_{3}\right)_{2} 0,01 \mathrm{~mol} \mathrm{~L}-1$, em $2 \mathrm{~g}$ de terra, agitadas por 1 e 24 horas em triplicata contendo concentrações crescentes de cada metal. Os resultados foram avaliados através do teste de comparação de curvas (Teste F). De maneira geral, as amostras agitadas por 24 horas adsorveram mais metais que as amostras agitadas por 1 hora. Pelos resultados, permite-se concluir que para as amostras de solo estudadas o tempo de agitação de 24 horas é o mais adequado para a obtenção da adsorção real dos metais estudados.
\end{abstract}

Palavras-chave: sorção, tempo de equilíbrio, metais pesados.

\section{ABSTRACT \\ INFLUENCE OF EQUILIBRIUM TIME IN Cd, Cu, Ni e Zn ADSORPTION ON SLUDGE-AMENDED SOIL SAMPLES}

The objective of the present paper is to evaluate the influence of equilibrium time in metal adsorption on sludge-amended soil samples $(0-0,2 \mathrm{~m})$ collected in a sewage sludge trial. Soil samples $(2.00 \mathrm{~g})$ were shaked during 1 and $24 \mathrm{~h}$ with $20 \mathrm{~mL}$ of $\mathrm{Ca}\left(\mathrm{NO}_{3}\right)_{2}$ 0,01 mol L-1 solution containing increasing concentrations of $\mathrm{Cd}, \mathrm{Cu}, \mathrm{Ni}$ and $\mathrm{Zn}$. Adsorption isotherms for 1 and $24 \mathrm{~h}$ were compared using the $\mathrm{F}$ test. In general greater amounts of metals were adsorbed when a 24 h-shaking time was used except for cadmium in sludge-amended sample. It is recommended a 24-h shaking period for equilibrium.

Key words: sorption; equilibration time; heavy metals.

$\left({ }^{1}\right)$ Recebido para publicação em 18 de dezembro de 2006 e aceito em 11 de julho de 2006.

$\left({ }^{2}\right)$ Centro de Solos e Recursos Ambientais do Instituto Agronômico de Campinas (IAC), Caixa Postal 28, $13012-970$ - Campinas SP). E-mail: evmellis @iac.sp.gov.br $\quad\left(^{*}\right)$ Autor correspondente.

$\left({ }^{3}\right)$ Departamento de Química, Escola Superior de Agricultura “Luis de Queiroz", Universidade de São Paulo, Escola Superior de Agricultura Luiz de Queiroz, Caixa-Postal: 09, 13418-900 Piracicaba (SP). E-mail: aarodell@esalq.usp.br 


\section{Introdução}

O tempo de agitação suficiente para completar o equilíbrio de adsorção dos íons metálicos é assunto que, embora bastante estudado, ainda permite divergências entre a comunidade científica. Segundo JORDÃo et al. (2000), o tempo de agitação para completar o equilíbrio de adsorção dos metais varia para diferentes tipos de solo e com o $\mathrm{pH}$.

Diversos autores estudaram a respeito do assunto, concluindo que o tempo ideal de agitação está entre 1 e 24 horas (SOARES et al., 2004; RAGHUPATHI e VASUKi ,1993; Jarvis, 1981; HARTer, 1984). Alguns autores recomendam a agitação por 24 horas para garantir o estabelecimento do equilíbrio de adsorção (Petruzelli et al., 1985). Na maioria dos trabalhos de adsorção em solos tropicais publicados, constata-se que se tem utilizado este tempo de agitação (SOARES, 2004; Mellis et al., 2004; Silveira et al. 2003, Dias et al., 2001; CASAGrande et al. 2004; Alleoni, et al., 2005; VeGA et al., 2006). Porém, nos últimos anos, alguns autores adotaram o tempo de agitação de 1 hora para se estudar a adsorção de metais em solos tropicais (Moreira, 2004; Fontes e Gomes, 2003). Embora a utilização deste tempo de agitação facilite a operacionalização dos estudos de adsorção em batelada, nenhum dos autores citados acima verificou em seus trabalhos se o equilíbrio de adsorção atingido no tempo de 1 hora é o mesmo obtido em 24 horas. Assim, o objetivo deste trabalho foi avaliar o efeito dos tempos de agitação de 1 e 24 horas no equilíbrio de adsorção em solo natural e tratado com lodo de esgoto.

\section{Material e Métodos}

As amostras que serviram de base para o estudo foram coletadas em março de $2005 \mathrm{em}$ um experimento onde, desde 1999, foram efetuadas cinco aplicações sucessivas de diferentes doses de lodos de esgoto na cultura do milho, instalado em Latossolo Vermelho Distroférrico (textura argilosa), localizado no Campo Experimental da Embrapa Meio Ambiente, em Jaguariúna (SP).

As amostras foram coletadas na superfície (0$0,2 \mathrm{~m}$ ), em parcelas que não receberam lodo de esgoto (testemunha) e nas parcelas que receberam em média 20 toneladas/aplicação de lodo oriundo da Estação de Tratamento de Esgoto de Barueri (SP). Tais parcelas foram escolhidas com o intuito de se definir qual o melhor tempo de agitação em situações contrastantes, baixos teores (testemunha) e altos teores de metais (solo com lodo de Barueri).

Após serem secas em estufa a $60^{\circ} \mathrm{C}$ até massa constante, as amostras foram devidamente preparadas para caracterização química (RAIJ et al., 2001). Os teores totais de $\mathrm{Cd}, \mathrm{Cu}, \mathrm{Ni}$ e $\mathrm{Zn}$ (Tabela 1) foram determinados pelo método SW 846-3051 (ЕpA, 1994).

Os estudos de adsorção seguiram o método de batelada (batch) de laboratório, (HAITcher e NAIDU, 2001), empregando-se como eletrólito suporte a solução de $\mathrm{Ca}\left(\mathrm{NO}_{3}\right)_{2}$ 0,01 $\mathrm{mol} \mathrm{L}^{-1}$ (relação 1:10) em substituição a solução $\mathrm{NaNO}_{3} 0,01 \mathrm{~mol} \mathrm{~L}^{-1}$, pois esta última provocou a dispersão da argila nas amostras tratadas com as concentrações mais altas dos metais.

As concentrações iniciais das soluções postas em contato com as amostras de solo, baseadas nos valores orientadores da Cetesb (2006), foram: 0; 0,1; 0,$5 ; 1,0 ; 1,5 ; 3,0 ; 4,0 ; 10 ; 18 \mathrm{mg} \mathrm{L}^{-1}$ de Cd como $\mathrm{Cd}\left(\mathrm{NO}_{3}\right)_{2} 4 \mathrm{H}_{2} \mathrm{O} ; 0 ; 5 ; 10 ; 25 ; 50 ; 70 ; 100 ; 140 ; 280 \mathrm{mg}$ $\mathrm{L}^{-1}$ de $\mathrm{Cu}$ como $\mathrm{Cu}\left(\mathrm{NO}_{3}\right)_{2} 2,5 \mathrm{H}_{2} \mathrm{O} ; 0 ; 1 ; 3 ; 5 ; 10 ; 20$; $30 ; 40 ; 60 \mathrm{mg} \mathrm{L}^{-1}$ de $\mathrm{Ni}$ como Ni $\left(\mathrm{NO}_{3}\right)_{2} 6 \mathrm{H}_{2} \mathrm{O} ; 0 ; 6 ; 10$; 30; 50; 100; 150; 300; $500 \mathrm{mg} \mathrm{L}^{-1}$ de $\mathrm{Zn}$ como $\mathrm{Zn}\left(\mathrm{NO}_{3}\right)_{2}$.

Para determinar a quantidade de metais adsorvidos, amostra de 2,00 g de terra fina seca em estufa foi agitada em agitador horizontal a 100 oscilações por minuto em tubos de polietileno com 20 $\mathrm{mL}$ de solução de $\mathrm{Ca}\left(\mathrm{NO}_{3}\right)_{2} 0,01 \mathrm{~mol} \mathrm{~L}^{-1}$, contendo diferentes concentrações de metais. Foram avaliados os tempos de agitação de 1 hora e 24 horas, em triplicata.

Os metais na solução de equilíbrio foram determinados por espectrofotometria de emissão atômica em plasma (ICP-AES). As quantidades de metal retido pela fase sólida (S) foram determinadas utilizando-se a seguinte expressão: $S=\left(C_{0}-C_{e}\right) F D$, em que $C_{0}$ é a concentração de metal da solução colocada em contato com o solo, $\mathrm{C}_{\mathrm{e}}$ é a concentração de metal na solução após a agitação e FD é fator de diluição (FD = $20 \mathrm{~mL}$ de solução/2 $\mathrm{g}$ de terra $=10$ ).

Tabela 1. Atributos químicos das amostras sem lodo (T) e com lodo de esgoto da ETE de Barueri (LB)

\begin{tabular}{|c|c|c|c|c|c|c|c|c|c|c|c|c|c|c|c|}
\hline Amostra & $\begin{array}{c}\mathrm{pH} \\
\mathrm{CaCl}_{2}\end{array}$ & $\mathrm{MO}$ & $\mathrm{P}$ & $\mathrm{K}$ & $\mathrm{Ca}$ & $\mathrm{Mg}$ & $\mathrm{H}+\mathrm{Al}$ & $\mathrm{Al}$ & SB & СТC & $\mathrm{V}$ & $\mathrm{Cd}^{*}$ & $\mathrm{Cu}^{*}$ & $\mathrm{Ni}^{*}$ & $\mathrm{Zn}$ * \\
\hline & & \multicolumn{2}{|c|}{$-\mathrm{g} \mathrm{dm}^{-3}-$} & \multicolumn{7}{|c|}{$\mathrm{mmol}_{\mathrm{c}} \cdot \mathrm{dm}^{-3}$} & $(\%)$ & \multicolumn{4}{|c|}{$\mathrm{mg} \cdot \mathrm{kg}^{-1}$} \\
\hline Testemunha & 5,3 & 26 & 6 & 0,5 & 33 & 15 & 30 & 1,0 & 48,5 & 78,5 & 62 & 0,90 & 13,8 & 1,8 & 18,1 \\
\hline S. c/ Lodo & 5,5 & 41 & 221 & 0,6 & 67 & 22 & 34 & 1,0 & 85,6 & 123,6 & 72 & 0,70 & 81,0 & 25,8 & 204,6 \\
\hline
\end{tabular}

* Teores Totais. Testemunha - solo não tratado com lodo de esgoto. S. c/ Lodo - solo tratado com lodo de esgoto. 
Avaliou-se o efeito do tempo de agitação na adsorção dos metais por meio de comparação de curvas de adsorção, em toda a sua extensão, através do teste $\mathrm{F}$, determinando se elas poderiam ser consideradas iguais do ponto de vista estatístico. As isotermas estudadas foram inicialmente ajustadas para os modelos de Langmuir e Freundlich com o auxílio do programa CurveExpert 1.3 (Hyams, 2004), sendo este último escolhido para a comparação pelo teste $\mathrm{F}$ devido ao melhor ajuste apresentado em relação à curva obtida experimentalmente.

Definido o modelo a ser utilizado, ajustou-se cada um dos conjuntos de dados experimentais, obtidos no tempo de agitação de 1 hora e 24 horas, a este. Em seguida, os dois conjuntos de dados reunidos em um único conjunto 1 hora +24 horas foram ajustados ao mesmo modelo; em seqüência, efetuouse a análise de variância para cada ajuste.

Os valores das somas de quadrados e os graus de liberdade do resíduo foram totalizados, designando-os como SQ separados e GL separados. Para o conjunto 1 hora +24 horas designou-se a soma de quadrados do resíduo como SQ combinado e os graus de liberdade como GL combinado. Calculou-se então o valor de F através da seguinte expressão:

$$
F=\frac{\frac{(S Q \text { combinado }-S Q \text { separado })}{(\text { GL combinado }- \text { GL separado })}}{\frac{(\text { SQ separado })}{(\text { GL separado })}}
$$

Determinou-se o valor da probabilidade $\mathrm{P}$ correspondente. O número de graus de liberdade do numerador é igual a GL combinado - GL separado e para o denominador GL separado. O valor da probabilidade $\mathrm{P}$ testa a hipótese de nulidade de que não há diferença entre as curvas ajustadas para " 1 hora" e "24 horas" e que qualquer diferença seria devida ao acaso. Valores de F calculado maiores que o F tabelado indicam que as duas curvas a serem comparadas diferem entre si.

\section{Resultados e Discussão}

Para todas as amostras, as curvas de adsorção diferiram estatisticamente quanto ao tempo de agitação (1 e 24 horas) para todos os metais, tanto no solo tratado com lodo, como no solo onde o lodo de esgoto não foi aplicado (Tabela 2).

De maneira geral, o tempo de agitação de 24 horas proporcionou maior adsorção de metais que o de 1 hora, exceto para o $\mathrm{Cd}$ na amostra tratada com lodo (Figura 1). Neste caso, as amostras agitadas por 1 hora adsorveram em torno de $5 \%$ a mais o metal que as amostras agitadas por 24 horas. SOARES et al. (2004), estudando a influência do tempo de agitação na adsorção de metais por vermicomposto de esterco bovino, verificaram que a adsorção de Co e $\mathrm{Zn}$ aumentou até 15 horas de agitação, decrescendo até 20 horas, em virtude da saturação do adsorbato, evidenciando o processo de dessorção. Em seguida, o sistema atingiu o equilíbrio, caracterizando a adsorção real dos metais no vermicomposto. Esses mesmos autores afirmam que o vermicomposto comporta-se, no que diz respeito à adsorção de metais, semelhantemente a solos com alto teor de matéria orgânica, como a amostra tratada com lodo estudada no presente trabalho (Tabela 1). Diante dessas observações, admite-se que o Cd tenha comportamento semelhante ao $\mathrm{Zn}$ e Co no vermicomposto, sendo, portanto mais seguro adotar o tempo de agitação de 24 horas nos estudos de adsorção em solos tratados com lodo de esgoto. Na amostra sem lodo, a diferença entre o Cd adsorvido após 24 horas de agitação foi por volta de $15 \%$ maior que nas agitadas por 1 hora nas doses mais baixas $\left(0-3 \mathrm{mg} \mathrm{L}^{-1}\right)$, e de $10 \%$ nas doses altas $\left(4-18 \mathrm{mg} \mathrm{L}^{-1}\right)$.

Tabela 2. Valores de F calculado para a comparação das curvas de adsorção em função do tempo de agitação

\begin{tabular}{lcccc}
\hline \multirow{2}{*}{ Tratamentos } & \multicolumn{4}{c}{ Metais $\left(^{1}\right)$} \\
\cline { 2 - 5 } & $\mathrm{Cd}$ & $\mathrm{Cu}$ & $\mathrm{Ni}$ & $\mathrm{Zn}$ \\
\hline Testemunha & $16,47^{*}$ & $3,26^{*}$ & $237,15^{*}$ & $4,39^{*}$ \\
Solo com Lodo & $10,32^{*}$ & $9,74 *$ & $120,75 *$ & 12,31 * \\
\hline
\end{tabular}

$\left.{ }^{1}\right)$ Para o caso de F $>1$ : F calculado $>$ F tabelado $(3,19)$ as curvas diferem entre si a $5 \%$ de probabilidade $(P=0,05)$.

$\mathrm{O} \mathrm{Cu}$, apesar da diferença significativa entre as curvas de adsorção nas duas amostras estudadas, foi o elemento com menor variação na adsorção em vista do tempo de agitação. Tanto na amostra sem lodo, como na tratada com o resíduo, até a dose de $50 \mathrm{mg}$ $\mathrm{L}^{-1}$ praticamente todo o $\mathrm{Cu}$ adicionado foi adsorvido, independentemente do tempo de agitação. A partir dessa dose, a adsorção no tempo de 24 horas foi maior que no tempo de 1 hora de agitação na amostra que não recebeu lodo. No solo tratado com lodo, essa diferença na adsorção devido ao tempo de agitação foi inferior a $5 \%$. Esse fato demonstra a alta afinidade dos constituintes do solo, principalmente a matéria orgânica com este elemento. Conforme JorDão et al. (2000), o tempo de agitação para completar o equilíbrio de adsorção do Cu varia entre 1 e 24 horas para diferentes tipos de solo. SOARES et al. (2004) verificaram que para vermicomposto de esterco bovino o equilíbrio é alcançado em torno de 20 horas, o que condiz com os resultados constantes deste trabalho. 

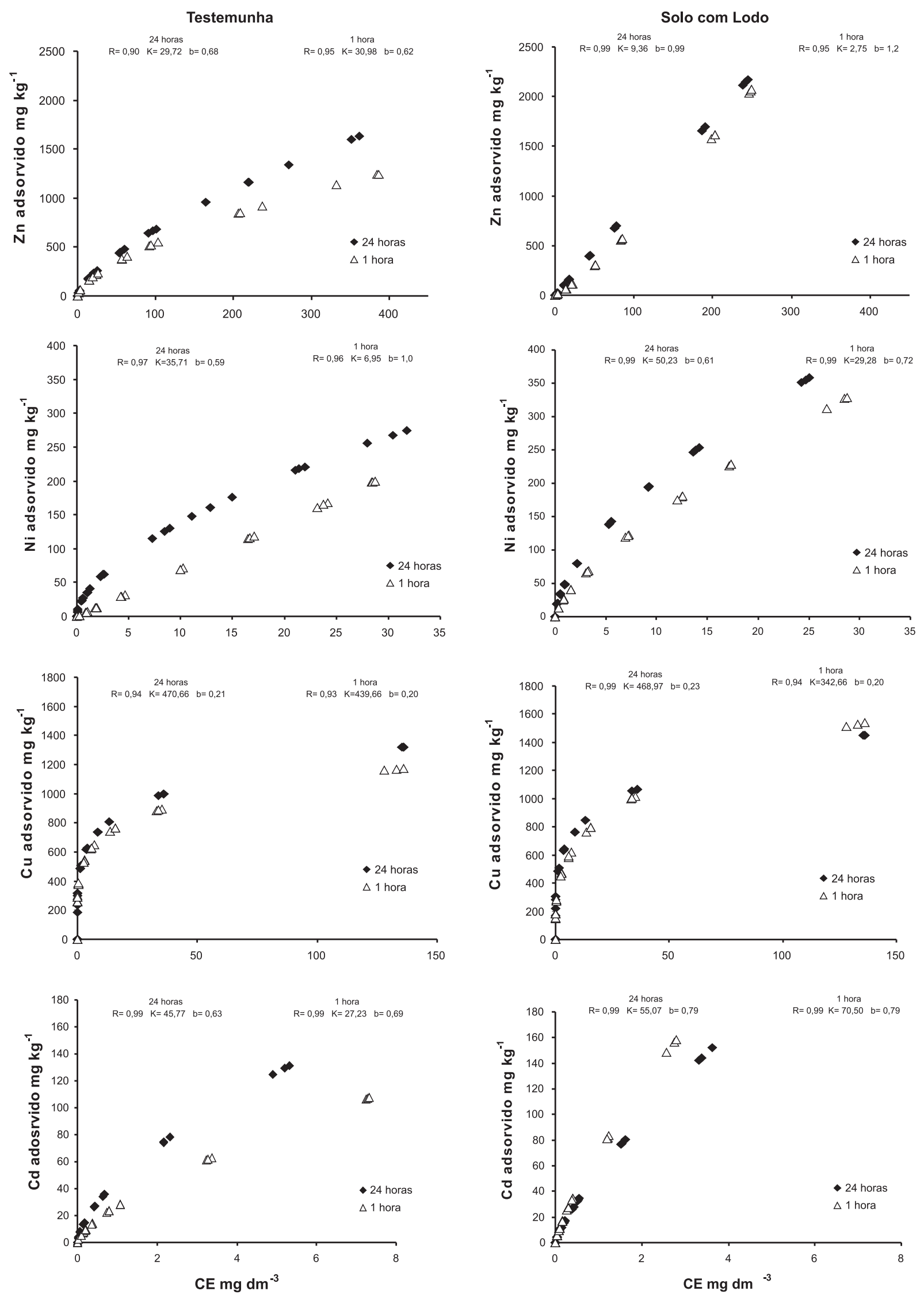

Figura 1. Isotermas de adsorção de $\mathrm{Cd}, \mathrm{Cu}, \mathrm{Ni}$ e $\mathrm{Zn}$ em função dos tempos de agitação (24 h e 1 h) em um Latossolo Vermelho Distroférrico não tratado e tratado com lodo de esgoto, ajustadas pelo modelo de Freundlich. 
Já a adsorção de Ni promoveu aumento médio de $15 \%$ nas doses inferiores a $30 \mathrm{mg} \mathrm{L}^{-1}$ no solo sem adição de lodo com a agitação por 24 horas. $\mathrm{Na}$ amostra tratada com lodo ocorreu a mesma tendência, porém nas doses mais elevadas do metal, 40 e $60 \mathrm{mg}$ $\mathrm{L}^{-1}$, o aumento de adsorção foi superior ao observado para as mesmas doses no solo sem lodo. MeluIs et al (2000) verificaram que quanto maior o tempo de contato do Ni com o solo, maior é a adsorção do metal.

O tempo de agitação também influenciou a adsorção de Zn. Tanto na amostra de solo tratado com lodo, como naquela que não recebeu aplicação de lodo houve aumento significativo da adsorção com o aumento do tempo de agitação. No solo sem lodo o incremento na adsorção de $\mathrm{Zn}$ provocado pelo aumento do tempo de agitação de 1 hora para 24 horas foi, em média, $7 \%$. Na amostra tratada com lodo observou se um decréscimo gradativo nas diferenças das porcentagens de adsorção em função do tempo de agitação, variando de $13 \%$ na menor dose até $2 \%$ na maior dose aplicada. Parveen et al. (1994) constataram que a biodisponibilidade de $\mathrm{Zn}$ em solos tratados com lodo de esgoto diminui com o aumento de $\mathrm{pH}$ e com o tempo de contato.

\section{CONCLUSÃO}

Pelos resultados, permite-se concluir que para as amostras de solo estudadas, o tempo de agitação de 24 horas é o mais adequado para a obtenção da adsorção real dos metais estudados.

\section{REFERÊNCIAS}

ALLEONI, L. R.F.; IGLESIAS, C.S.M.; MELLO, S.C.; CAMARGO, O.A.; CASAGRANDE, J.C.; LAVORENTI, N.A. Atributos do solo relacionados à adsorção de cádmio e cobre em solos tropicais. Acta Scientarum Agronomica, Maringá, v.27, n.4, p.729-737, 2005.

CASAGRANDE, J.C.; JORDÃO, C.B.; ALLEONI, L.R.F; CAMARGO, O.A. Copper desorption in a variable eletrical charge soil. Scientia Agricola, Piracicaba, v.61, n.2, p. 196-202, 2004.

COMPANHIA DE TECNOLOGIA DESANEAMENTO BÁSICO (CETESB), Relatório de Estabelecimento de Valores Orientadores para Solos e Águas Subterrâneas no Estado de São Paulo.Valores Orientadores para Solos e Águas Subterrâneas no Estado de São Paulo. Dispopnível em: http:/ /www.cetesb.sp.gov.br/solo/valoresorientadores. Acesso em 5 de dez. 2006.

DIAS, N.M.P; ALLEONI, L.R.F.; CASAGRANDE, J.C.; CAMARGO, O.A. Adsorção de cádmio em dois Latossolos ácricos e um Nitossolo. Revista Brasileira de Ciência do Solo, Viçosa, v.25, p.297-304, 2001.
ENVIRONMENTAL PROTECTION AGENCY-EPA. Method 3051 - Microwave assisted acid digestion of sediments, sludges, soils, and oils. Washington, DC, 1994. p.14.

FONTES, M.P.F.; GOMES, P.C. Simultaneous competitive adsorption of heavy metals by the mineral matrix of tropical soils. Applied Geochemistry, Aberystwyth, v.18, p.795-804, 2003.

HAITCHER, R.D.; NAIDU, R. An assessment of environmental and solution parameter impact on trace-metal sorption by soils. Soil Science Society of America Journal, Madison, v.65, n.3, p.597-612, 2001.

HARTER, R. Curve-fit errors in Langmuir adsorption maxima. Soil Science of America Journal, Madison, v.48, p.749-752, 1984.

HYAMS, D. CurveExpert 1.37. Microsoft Corporation, 1993. Disponível em: http:/ / www.ebicom.net. Acesso em: 5 set 2006.

JARVIS, S.C. Copper sorption by soils at low concentrations and relation to uptake by plants. Journal of Soil Science, London, v.32, p.257-269, 1981.

JORDÃO, C.P.; ALVES, N.M.; PEREIRA, J.L.; BELLATO, C.R. Adsorção de íons $\mathrm{Cu}^{2+}$ em Latossolo Vermelho-Amarelo húmico. Química Nova, São Paulo, v.23, n.1, p.5-11, 2000.

MELLIS, E.V.; CASAGRANDE, J. C. Adsorção/dessorção de níquel em um Latossolo Amarelo ácrico do Estado de São Paulo. In: REUNIÃO BRASILEIRA DE FERTILIDADE DOSOLO E NUTRIÇÃO DE PLANTAS, 24., 2000, Santa Maria. Resumos... Santa Maria: Sociedade Brasileira de Ciência do Solo, 2000. (CD-ROM)

MELLIS, E.V.; CRUZ, M.C.P.; CASAGRANDE, J.C. Nickel adsoption by soils in relation to $\mathrm{pH}$, organic matter and iron oxides. Scientia Agricola, Piracicaba, v. 61, n. 2, p. 190195, 2004.

MOREIRA, C.S. Adsorção competitiva de cádmio, cobre, níquel e zinco em solos. 2004. 108p. Dissertação (Mestrado)- Escola Superior de Agricultura "Luiz de Queiroz", Universidade de São Paulo, Piracicaba, 2004.

PARVEEN, Z.; EDWARDS, A.C.; CRESSER, M.S. Redistribution of zinc from sewage sludge applied to a range of contrasting soils. The Science of the Total Environment, Amsterdam, v. 155, p. 161-171, 1994.

PETRUZZELLI, G.; GUIDI, G.; LUBRANO, L. Ionic strength effect on heavy metal adsorption by soil. Communications in Soil Science and Plant Analysis, New York, v. 16, p. 971986, 1985.

RAIJ, B. van; ANDRADE, J.C.; CANTARELLA, H.; QUAGGIO, J.A. Análise química para avaliação da fertilidade de solos tropicais. Campinas: Instituto Agronômico, 2001. 285p.

RAGHUPATHI, H. B., VASUKI, N. Copper adsorption of some soils of north Karnataka. Journal of the Indian Society of Soil Science, New Delhi, v. 41, p. 70-74, 1993. 
SILVEIRA, M.L.A.; ALLEONI, L.R.F. Copper adsorption in tropical Oxisols. Brazilian Archives of Biology and Technology, Curitiba, v. 46, p. 529-536, 2003.

SOARES J.P., SOUZA J.A de, CAVALHEIRO E.T.G. Caracterização das amostras comerciais de vermicomposto de esterco bovino e avaliação da influência do $\mathrm{pH}$ e do tempo na adsorção de Co (II), Zn (II) e Cu (II). Química Nova, São Paulo, v. 27, n. 1, p.5-9, 2004.

SOARES, M.R. Coeficiente de distribuição $\left(K_{\mathrm{D}}\right)$ de metais pesados em solos do Estado de São Paulo. 2004. 202p. Tese (Doutorado) - Escola Superior de Agricultura "Luiz de Queiroz", Universidade de São Paulo, Piracicaba, 2004.

VEGA, F. A.; COVELO, E. F.; ANDRADE, M. L. Competitive sorption and desorption of heavy metals in mine soils: Influence of mine soil characteristics. Journal of Colloid and Interface Science, New York, v. 298, p. 582-592, 2006. 